\title{
OPEN Mathematical models for thermionic emission current density of graphene emitter
}

\author{
Olukunle C. Olawole ${ }^{1 凶}$, Dilip K. De ${ }^{2}$, Sunday O. Oyedepo ${ }^{3} \&$ Fabian I. Ezema ${ }^{4,5,6,7}$
}

In this study, five mathematical models were fitted in the absence of space charge with experimental data to find a more appropriate model and predict the emission current density of the graphene-based thermionic energy converter accurately. Modified Richardson Dushman model (MRDE) shows that TEC's electron emission depends on temperature, Fermi energy, work function, and coefficient of thermal expansion. Lowest Least square value of $S=\sum\left(J_{t h}-J_{\text {exp }}\right)^{2}=0.0002 \mathrm{~A}^{2} / \mathrm{m}^{4}$ makes MRDE most suitable in modelling the emission current density of the graphene-based TEC over the other four tested models. The developed MRDE can be adopted in predicting the current emission density of twodimensional materials and also future graphene-based TEC response.

As a family member of two-dimensional (2D) materials, graphene remains an electrode of choice in the heart of researchers in harvesting electricity via thermionic and photo-enhanced thermionic energy converter due to its unmatched potentials ${ }^{1-8}$. Because of its unmatched prospects in electronics, thermal expansion, optical and mechanical properties, ease of tune-ability of its work function that is seen to operate in the $2 \mathrm{D}$ world ${ }^{9-15}$ and graphene's tolerance of high temperature ${ }^{16}$. Recently the mechanical properties were experimentally proven to use also in the 3D world ${ }^{12,17}$. In principle, Fig. la shows how the emitted electrons (working fluid) can be induced through thermal ${ }^{6,7,18-20}$, photo ${ }^{4-6,21,22}$, secondary ${ }^{6,23}$ and field emission ${ }^{6,24-27}$. The thermionically excited electrons work as the working fluid above the potential interface barrier and, after that, is collected at the anode of the TEC. Fundamentally, a TEC setup comprises a cathode (B), an anode (A), a conducting wire, and a load (Fig. 1d). And for this to perform optimally, the work function of electrode B must be greater than the work function of anode $A$; the temperature of $B$ must be higher than the temperature of $A$. The distance between $\mathrm{A}$ and $\mathrm{B}$ must be of the order of micrometer to minimize the effects of space charge and thus, for the emitted electron to reach the collector and in the absence of space charge. However, when the electrodes in Fig. $1 \mathrm{~b}$ are not electrically connected, its vacuum energy remains the same. Consequently, when electrodes in Fig. 1c are electrically connected, the alignment of chemical potential is noticed due to electrons migration from electrode A to electrode B. In addition, the presence of chemical potential in Fig. 1c creates a potential difference. Moreover, this potential difference in Fig. 1c stops further migration of electrons from anode to cathode. Nevertheless, as soon as the external load is attached to the setup in the presence of continuous supply of thermal energy from sunlight as depicted in Fig. 1d, the electrons at the cathode would have sufficient energy to overcome a surface barrier, migrate to the anode and drive electricity through the TEC.

Despite enormous research in TEC, the TEC technology's potential in generating electricity is still being hindered due to high material work function and space charge related problems $s^{7,28-30}$. This has led to the unnatural death of technology in the $90 \mathrm{~s}$. Nevertheless, a recent revival of interests in thermionic energy converters is attributed to the emerging nanomaterials and ever-growing technology in the twenty-first century ${ }^{5,6,8}$. The technologies that are primarily of interest for applying graphene-based TEC are graphene's growth on solid tungsten and silicon carbide substrates ${ }^{16,31-33}$ and ease of work function engineering of graphene surface ${ }^{13,15,34-36}$.

Theoretically, researchers have seen green light about the terrestrial applications of TEC instead of space applications through the usage of nanomaterials as electrodes in TEC. In harnessing a graphene-based TEC's

\footnotetext{
${ }^{1}$ Department of Physics, Covenant University, Ota, Ogun State, Nigeria. ${ }^{2}$ Sustainable Green Power Technologies, San Antonio, TX, USA. ${ }^{3}$ Department of Mechanical Engineering, Covenant University, Ota, Ogun State, Nigeria. ${ }^{4}$ UNESCO-UNISA Africa Chair in Nanosciences-Nanotechnology, PO Box 392, Pretoria, South Africa. ${ }^{5}$ Nanosciences African Network (NANOAFNET), iThemba LABS-National Research Foundation, PO Box 722, Somerset West, Western Cape Province, South Africa. ${ }^{6}$ Department of Physics and Astronomy, University of Nigeria, Nsukka, Nigeria. ${ }^{7}$ Africa Centre of Excellence for Sustainable Power and Energy Development (ACE-SPED) University of Nigeria, Nsukka, Nigeria. ${ }^{\bowtie}$ email: olukunle.olawole@covenantuniversity.edu.ng
} 


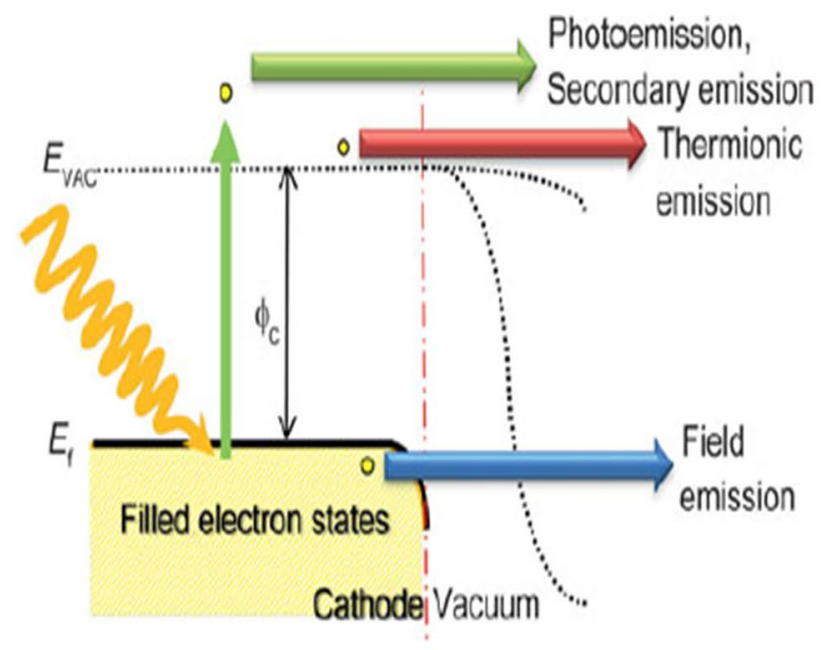

(a)

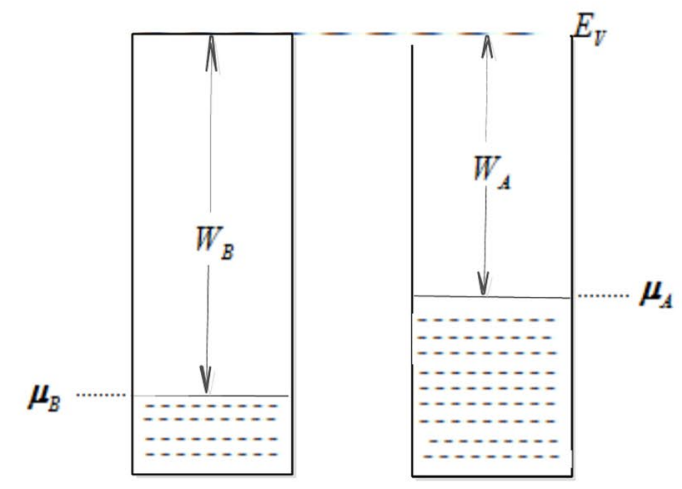

(b)

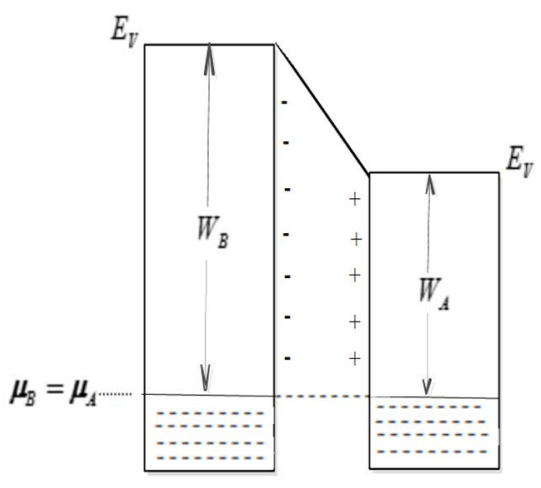

(c)

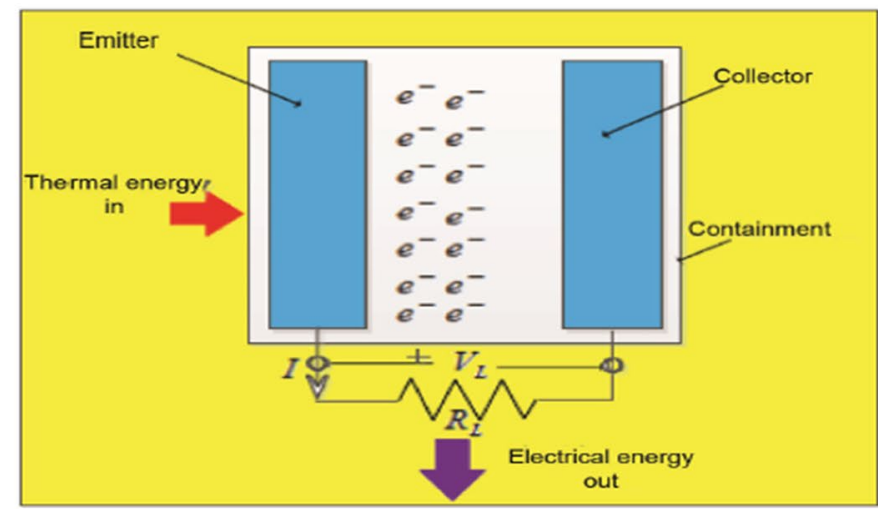

(d)

Figure 1. (a) Various mechanisms of producing electron emission ${ }^{6,17,30}$. (b) Unconnected cathode and anode ${ }^{7}$. Reprinted (a) with permission from Trucchi, D. M. \& Melosh, N. A. Electron-emission materials: Advances, applications, and models. MRS Bull. (2017) https://doi.org/10.1557/mrs.2017.142. Copyright (2021) by the SpringerNature. (c) Connected cathode and anode ${ }^{7}$. (d) Thermionic energy converter set up ${ }^{7}$.

hidden electric power generation potential, specific parameters such as power output, thermal exchange, heat removal rate, and efficiency must be accurately modelled with some other TEC parameters.

Power output of graphene-based TEC. The formation of the potential barrier $\frac{\left(W_{e}-W_{c}\right)}{e}$ (in the absence of space charge) in Fig. 1c is explained in detail in references ${ }^{7}$ and ${ }^{8}$. Once electrons are emitted thermionically at the emitter by overcoming the barrier due to its work function), the electrons find an electric field between the emitter and the anode due to the potential barrier $\frac{\left(W_{e}-W_{c}\right)}{e}$. These electrons would deliver power at the load $P_{0}$ as in Eqs. (1-2) (Fig. 2).

The present study aimed at examining the power output of graphene-based TEC specifically in the absence of space charge:

$$
\begin{gathered}
P_{0}=\left(I_{e}-I_{c}\right) \frac{\left(W_{e}-W_{c}\right)}{e} \\
P_{0}=\left(J_{e}-J_{c}\right) s\left(\frac{W_{e}-W_{c}}{e}\right)
\end{gathered}
$$

where $I_{e}$ is the thermionic current of the emitter, $I_{c}$ is the thermionic current collector, $T_{e}$ is the temperature of the emitter, $T_{c}$ is the temperature of the collector. In addition, $W_{e}$ is the of emitter's work function, $W_{c}$ denotes 


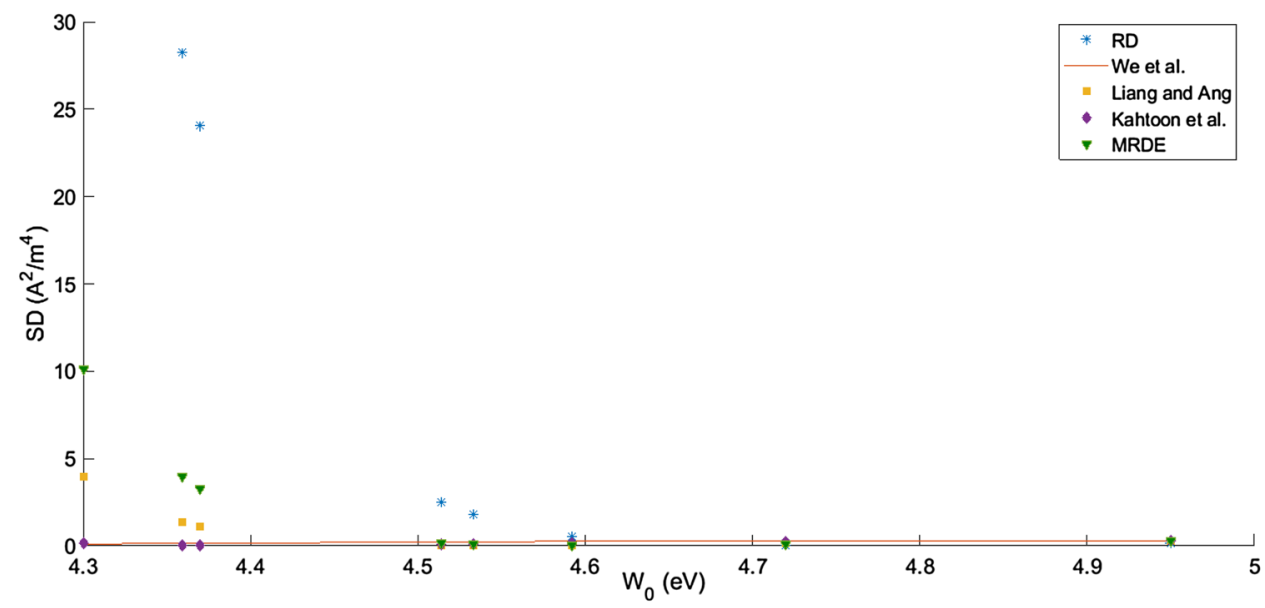

Figure 2. $S D\left(\mathrm{~A}^{2} / \mathrm{m}^{4}\right)$ against the work function $(\mathrm{eV})$ of five models in TEC.

collector's work function, $e$ typifies electronic charge, and the power output is represented by $P_{0}$. However, $J_{e}$ is the emitter's current density, $J_{c}$ represents the collector's current density and $s$ connotes the emitter's surface area.

Fundamental equation for energy balance in TEC. So far, modelling a TEC has neglected heat radiation losses, affecting the efficiency calculation seriously, as shown by Olawole and $\mathrm{De}^{7}$. Incorrect modelling of efficiency of a TEC energy conservation which was not appropriately modelled except in Refs. ${ }^{737}$ must be taken fully into account. This is given for a solar TEC by Eq. (3) ${ }^{38-40}$.

$$
I_{0}(S-s)=\left[\left(\frac{J_{e} s\left(W_{e}+2 k_{B} T_{e}\right)}{e}-\frac{J_{\mathcal{c}} s\left(W_{c}+2 k_{B} T_{c}\right)}{e}\right)+\left[\sigma s\left(T_{e}^{4}-T_{a}^{4}\right)\right]+\left[\sigma s\left(T_{e}^{4}-T_{c}^{4}\right)\right]\right]
$$

where $I_{0}$ is the solar irradiance, $S$ connotes the area of the parabolic concentrator, $\mathrm{s}$ is known as the emitter's area and $\sigma$ stands for Stefan Boltzmann's constant. Alternately, where the heat source is a burner or some other device, $I_{0}(S-s)$ is the incident total heat flux at the emitter.

Therefore, the first term on the right side of Eq. (3) $J_{e} s\left(W_{e}+2 k_{B} T_{e}\right) / e$ shows the total energy acquired by ejected electrons at the emitter. Likewise, the second term on the right hand of Eq. (3) $\left[J_{c} s\left(W_{c}+2 k_{B} T_{c}\right) / e\right]$ depicts the total energy electrons at collector absorbed. Furthermore, $\left[J_{e}\left(2 k_{B} T_{e}\right) / e\right]$ denotes heat energy imparted on the collector by the emitted electrons from the cathode. Besides, $\left[J_{c}\left(2 k_{B} T_{c}\right) / e\right]$ is the thermal energy the electrons emitted from an anode imparted on the cathode.

Rate of thermal energy removal in TEC. In principle, the temperature of the collector plate rises fast due to the bombardment of the emitted electrons from the cathode. The thermal removal rate will be modelled with Eq. (4):

$$
Q_{r}=\left[\left(\frac{J_{e}\left(2 k_{B} T_{e}\right)}{e}-\frac{J_{c}\left(2 k_{B} T_{c}\right)}{e}+\sigma\left(T_{e}^{4}-T_{c}^{4}\right)\right) s\right]
$$

where $Q_{r}$ is the thermal removal rate from the collector to keep its temperature at $\mathrm{T}_{\mathrm{c}}$ in the TEC system.

The efficiency of TEC. The efficiency of graphene-based solar TEC can be modelled by Eq. (5):

$$
\eta=\frac{\left(W_{e}-W_{c}\right)\left(J_{e}-J_{c}\right) s}{I_{0}(S-s) e}
$$

where $I_{0}(S-s)$ is the total solar power incident on the emitter. From Eqs. (3)-(5) can be deduced the influence of the parameters, $I_{o}, \mathrm{~S}, \mathrm{~s}, \mathrm{~T}_{\mathrm{e}}$ and $\mathrm{T}_{\mathrm{c}}, W_{e}, W_{c}$ on $\eta$. Thus, it is essential to correct $\mathrm{J} \mathrm{vs}$. $\mathrm{T}$ for nanomaterials to model a TEC. Equation (5) stands universal and applies to all TEC ${ }^{7,8,37,41,42}$. Graphene also has been found suitable for thermionic energy conversion ${ }^{7,28,37,40,43-48}$. Thus arises the importance of correct modelling of J vs. T for proper modelling of the efficiency of solar and other types of thermionic energy converters. For solar TEC Eq. (3) is found useful ${ }^{7,37}$. It is to be noted that for proper modelling of $\mathrm{J}$ vs $\mathrm{T}$, the dependence of $\mathrm{W}$ on $\mathrm{T}$ must be taken into account. This aspect has been discussed earlier to some extent for TEC conversion of solar energy to generate electricity in future. More needs to be done, especially for nanomaterials.

The recent issue being raised in scientific communities is the fitness of the Richardson Dushman (RD) equation that was enacted for metals ${ }^{18,28,49}$ only in predicting and determining the current density of nano-thermionic energy ${ }^{6,20,47}$ converter. In response to the fitness of the RD equation, some scholars have affirmed the potency of the RD equation in determining the current density of the emitters and collectors in nano-thermionic engines ${ }^{58-60}$. At the same time, some researchers opined that using macroscopic RD law to investigate a nano-thermionic 
energy converter (NTEC) is against the physics of nanoscience and nano-engineering ${ }^{7,20,37,50-52,61}$. To resolve this challenge, the experimental results of Zhu et al. ${ }^{44}$ for J vs. T in graphene have been adopted to fit the various models and to find the most suitable model for predicting the accuracy of the current density of graphene-based thermionic energy converter.

\section{Methodology}

This study has acquired graphene's experimental thermionic emission (J vs. T) data from reliable and standard source $^{44}$. Consequently, the five theoretical models (Eqs. 36-40) were coded in MATLAB to fit the experimental current density of graphene-based TEC.

Richardson-Dushman model. Nobel Prize-winning Eq. (6) of Richardson-Dushman was explained on the theory of Sommerfeld for probing the mechanism of electron emission in electronic devices. Equation (6) opined that the current density of the thermionic converter is a subject of the work function and temperature of the metal ${ }^{49}$ :

$$
J=A_{0} T^{2} \exp \left(-\frac{W}{k_{B} T}\right)
$$

Typically, $A_{0}=\frac{4 \pi e m k_{B}^{2}}{h^{3}}$ denotes Richardson-Dushman value $1.2 \times 10^{6} \mathrm{Am}^{-2} \mathrm{~K}^{-2}, W$ stands for work a function, $\mathrm{T}$ is the temperature, $\mathrm{e}$ is the electronic charge, $\mathrm{m}$ is the electron mass, $\mathrm{h}$ typifies the Planck's constant, and $k_{B}$ connotes the Boltzmann constant.

Wei et al. and Kim and Lee model. Investigation of Wei et al. ${ }^{52}$ confirmed that Sommerfeld theory could have been a suitable model in predicting electron emission from the edge of two-dimensional (2D) materials like graphene. However, the current density from the edge is too small to be of any practical importance. Hence, the scientific thought of Wei et al. ${ }^{52}$ and Kim and Lee ${ }^{53}$ on the electron emission from the graphene sheet with atomic thickness is expressed as:

$$
J=A_{0} T^{3 / 2} \exp \left(-\frac{W}{k_{B} T}\right)
$$

where $A_{0}=\left[(m / 2)^{1 / 2}\left(k_{B} / \pi\right)^{3 / 2}(e) /(\hbar)^{2}\right]=2.2573 \times 10^{4} A \mathrm{~m}^{-2} \mathrm{~K}^{-3 / 2}$ is the Richardson-Dushman constant for graphene, $\mathrm{W}$ is the work function of the material, $\mathrm{T}$ is the temperature, and $k_{B}$ is the Boltzmann constant.

Moreover, a complexity in their current density versus temperature model when tunnelling effect is applied at a different energy level and time interval in examining the confinement of electrons in a potential barrier poses a fundamental question on the correctness of their theory. Also, this theory should be conceived in the heart and not in the practical term.

Liang and Ang model. The theoretical investigation of Liang et al. ${ }^{20}$ showed that the RD is a non-valid theory to examine the current emission density of graphene. Their study considered the in-plane mass of graphene electrons as a zero entity. Even though they thought the finite mass of electrons for motion perpendicular to the graphene surface, they got rid of this finite mass in the final equation using some questionable tricks. They assumed a temperature-independent work function. This allows their study to adopt a new Richardson Dushman constant of $115.8 \mathrm{Am}^{-2} \mathrm{~K}^{-3}$. On the contrary, Yoon et al. ${ }^{54}$ experiment revealed the non-massless nature of graphene when the estimated dynamical electron mass of graphene is found between $0.01 m_{e}$ to $0.024 m_{e}$, which raises doubt on Liang and Ang idea $^{20}$ in Eq. (8).

They did not consider the discrete nature of electron energy perpendicular to the graphene surface. In their theory, they put the lower limit of integration for the energy of emitted electron as $\phi$ whereas it should be $E_{F}+\phi$, which is the minimum energy of the electron which can be emitted. The energy of the electron at that point is $E_{F}+\phi$ and not just $\phi$.

Consequently, their findings opined that the current density of graphene-based TEC is dependent on a cube of its temperature and work function and Fermi energy, as shown in Eq. (38) ${ }^{20}$

$$
J=\frac{e k_{B}^{3} T^{3}}{\pi \hbar^{3} v_{F}^{2}} \exp \left(-\frac{W-E_{F}}{k_{B} T}\right)
$$

$A_{0}=\frac{e k_{B}^{3}}{\pi \hbar^{3} v_{F}^{2}}$ is the assumed Richardson Dushman constant for graphene. $=115.8 A m^{-2} K^{-3}$. Where $\mathrm{W}$ is the work function of the material, $E_{F}$ is the Fermi energy $v_{F}$ is the Fermi velocity, T is the temperature, $\mathrm{e}$ is the electronic charge, $\hbar$ is the reduced Planck's constant, and $k_{B}$ is the Boltzmann constant. In their Eq. (8), they have treated $E_{F}$ and $v_{F}$ as two separate items contradictory to Eq. (9):

$$
E_{F}=m_{c} V_{F}^{2}
$$

Khatoon Ansari and Ashraf model. Consequently, Khatoon et al. considered how to work function and Fermi energy are a function of temperature in ${ }^{50}$ : 


$$
J=\frac{e k_{B}^{3} T^{3}}{\pi \hbar^{3} v_{F}^{2}} \exp \left(-\frac{\left[(\phi(0)+\mu(0)(1-\alpha T))-(\mu(0) / 2 \ln 2)\left(T_{F} / T\right)\right]}{k_{B} T}\right)
$$

$A_{0}=\frac{e k_{B}^{3}}{\pi \hbar^{3} v_{F}^{2}}$ is an assumed Richardson Dushman constant for graphene and equals $115.8 A m^{-2} K^{-3}$ as in Liang and Ang model. Where $\phi(0)$ is the work function of the material at absolute zero temperature, $\alpha$ is the coefficient of thermal expansion, $\mu(0)$ is the Fermi energy at absolute zero temperature, $v_{F}$ is the Fermi velocity, $\mathrm{T}$ is the temperature, $T_{F}$ is the Fermi temperature, $\mathrm{e}$ is the electronic charge, $\hbar$ is the reduced Planck's constant, and $k_{B}$ is the Boltzmann constant.

De and Olawole model. De and Olawole remarked that three dimensional (3D) approach has the potential to model the mechanism of thermionic emission in $2 \mathrm{D}$ electrodes (graphene) based on the following conditions ${ }^{37}$ :

1. That Fermi energy $E_{F}(T)$, work function (W) and thermal expansion of $2 \mathrm{D}$ electrodes are a function of temperature.

2. Thermionic emission must be perpendicular to the graphene surface. For in-plane motion, the electrons must possess in-plane momentum components $\left(k_{x}, k_{y}\right)$. For emission normal to the z-plane, the electron must possess $k_{z}$ component of momentum. Thus, the electron must possess three components of momentum for thermionic emission from the 2-D graphene.

3. Re-filling of the vacant energy sites created by the emitted electrons at emitter with electrons, through back electrons from the anode to cathode.

4. z-component of graphene-based thermionic emission possesses momentum in the 3D world provided $W+E_{F} \leq\left|\frac{P_{z}^{2}}{2 m}\right| \leq \infty$.

5. It has also been noted that the generation of electricity through edge emission is impracticable ${ }^{52}$ as the current will be too small. In addition, the following scenarios that dictate the emissions of electrons from graphene along $\mathrm{z}$-direction need to be considered:

(a) Electrons in z-plane possess discrete energy levels as particles which may correspond either to an infinitely square well potential or a finite square well such that though the electron motion in the plane of graphene is massless corresponding to the electron dispersion relation: $E(q)= \pm V_{F}|q|$; however, it possesses finite mass for motion along the $\mathrm{z}$-direction. Electron emission takes place when it contains energy more significant than the work function of the material.

(b) Let's consider electron energy levels to be dictated by the finite square well. Then, tunnelling probability must be regarded as allowing emission to occur, especially when the energy of tunnelling electrons is higher than the work function of the confinement.

So far, no model has considered all the above realistic scenarios of dynamics of electron emission from the 2-D graphene, as it is too complicated to handle theoretically.

To avoid the complication associated with a near-perfect model as mentioned above, considering no. (v) De and Olawole assumed a 3D model (due to three momentum of the electrons) considering thermal expansion of the lattice and temperature dependence of work function. The 3D model of graphene is recently found to hold for mechanical properties of graphene ${ }^{12}$. They also considered the temperature dependence of work function. With these considerations, the Richardson Dushman equation was modified to model the current density of graphene in terms of Fermi energy and work function dependent on temperature t $^{7,8,37,45}$.

$$
J=A_{0} T^{2} \exp \left(-\left[W_{0}+\left(\left[r \alpha T+(1+r \alpha T)\left(\frac{\pi^{2}}{12}\right)\left(\frac{k_{B} T}{E_{F 0}}\right)^{2}\right] E_{F 0}+(1+r \alpha T)\left(\frac{7 \pi^{4}}{960}\right)\left(\frac{k_{B} T}{E_{F 0}}\right)^{4}\left(E_{F 0}\right)\right)\right] / k_{B} T\right)
$$

$A_{0}=\frac{4 \pi e m k_{B}^{2}}{h^{3}}$ is the Richardson-Dushman constant for graphene $=1.2 \times 10^{6} \mathrm{Am}^{-2} \mathrm{~K}^{-2}$. Where $\mathrm{W}_{0}$ is the work function of the material, $\mathrm{r}$ is the dimensionality of the material, such that $2 \mathrm{D}$ graphene, has a $\mathrm{r}$ value of 2 while carbon nanotube has a $\mathrm{r}$ value of $1, \mathrm{~T}$ is the temperature, $\alpha$ is the coefficient of thermal expansion, $\mathrm{e}$ is the electronic charge, $\mathrm{m}$ is the electron mass, $\mathrm{h}$ typifies the Planck's constant, $E_{F 0}$ is the Fermi energy, and $k_{B}$ is the Boltzmann constant.

\section{Results and discussion}

Table 1 depicts how different parameters $\left(W_{0}, E_{F 0}, V_{F}\right)$ affect the various least-square values for the five models. The least-square method is used to select work function, Fermi energy, and Fermi velocity that fits most of the thermionic emission current density data (experimental) of 2D materials (graphene) with the model's theoretical values the model could be the best.

Figure 3 shows the best fit of the experimental data of J vs. T with different models. Interestingly, the solid red line of Eq. (6) slightly fits the experimental black dotted points at $W_{0}=4.720 \mathrm{eV}$ with a least-square value of $0.0006 \mathrm{~A}^{2} / \mathrm{m}^{4}$. In addition, the yellow cross of Eq. (7) in Fig. 3 initially deviates slightly from the experimental black dotted points at $1620-1755 \mathrm{~K}$ and later fits at $1760-1795 \mathrm{~K}$. Its least-square value is $0.1170 \mathrm{~A}^{2} / \mathrm{m}^{4}$ with tuned $W_{0}=4.300 \mathrm{eV}$. Consequently, the magenta star of Eq. (8) in Fig. 3 fits the experimental black dotted points at $1620-1730 \mathrm{~K}$ and deviates fairly at $1740-1795 \mathrm{~K}$. Its least-square value is $0.0004 \mathrm{~A}^{2} / \mathrm{m}^{4}$ with tuned $W_{0}=4.533 \mathrm{eV}, E_{F 0}=0.083 \mathrm{eV}$ and $V_{F}=1 \times 10^{6} \mathrm{~m} / \mathrm{s}$. The cyan diamond of Eq. (9) in Fig. 3 deviates slightly from the experimental black dotted points from $1620 \mathrm{~K}$ up to $1795 \mathrm{~K}$ with a least-square value of $0.0053 \mathrm{~A}^{2} / \mathrm{m}^{4}$, $W_{0}=4.3700 \mathrm{eV}, E_{F 0}=0.083 \mathrm{eV}$ and $V_{F}=1 \times 10^{6} \mathrm{~m} / \mathrm{s}$. 


\begin{tabular}{|l|l|l|l|l|l|}
\hline Models & Year & $\mathbf{W}_{\mathbf{0}}(\mathbf{e V})$ & $\begin{array}{l}\mathbf{E}_{\mathbf{F} 0} \\
(\mathbf{e V})\end{array}$ & $\begin{array}{l}\mathbf{V}_{\mathbf{F}}(\mathbf{m} / \mathbf{s}) \\
\mathbf{1 0}^{\mathbf{6}}\end{array}$ & $\mathbf{S D}=\left(\mathbf{A}^{2} / \mathbf{m}^{4}\right)$ \\
\hline Richardson Dushman & 1928 & 4.720 & - & - & 0.0006 \\
\hline Wei et al & 2013 & 4.300 & & & 0.1170 \\
\hline Liang and Ang & 2015 & 4.533 & 0.083 & 1.000 & 0.0004 \\
\hline De and Olawole & 2018 & 4.592 & 0.203 & - & 0.0002 \\
\hline Kahtoon et al & 2018 & 4.370 & 0.083 & 1.000 & 0.0053 \\
\hline & & 4.514 & 0.083 & 2.49 & 0.0984 \\
\hline
\end{tabular}

Table 1. Influence of work function, Fermi energy, and Fermi velocity on the current density of graphene as dictated by the square difference (SD).

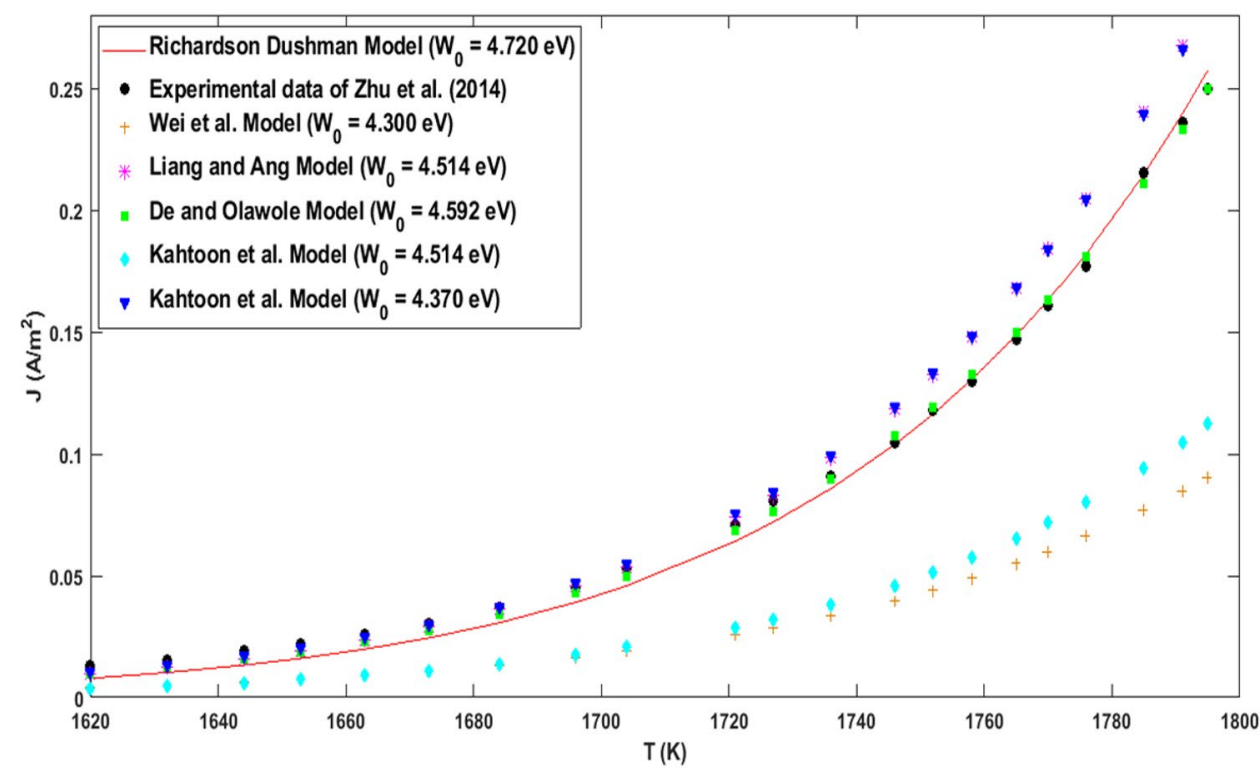

Figure 3. Best fitting of the theoretical thermionic emission current density of graphene with experimental data (black dotted points) for different models of graphene. The solid red line for Eq. (6) (RD model), yellow cross for Eq. (7) (Wei et al., model), magneta solid for Eq. (8) (Liang and Ang model), cyan diamond and blue triangular for Eq. (10) (Khatoon et al., model), and green square for Eq. (11) (De and Olawole model).

While the green square of Eq. (11) in Fig. 3 shows a good fit with the experimental black dotted points at $W_{0}=4.592 \mathrm{eV}, E_{F 0}=0.203 \mathrm{eV}$ which results in the least-square value of $0.0002 \mathrm{~A}^{2} / \mathrm{m}^{4}$. Statistically and graphically, the representation of Table 1 and Figs. 2 and 3 have shown that Eq. (11) modelled experimental data $^{44}$ of graphene accurately as its electron emission depends on temperature, Fermi energy, work function, and coefficient of expansion. That graphene's Fermi energy is temperature-dependent, precisely at $0 \mathrm{~K}$, which was ignored in Eqs. $(6-8,10)^{20}$.

We propose the fitting of De and Olawole model ${ }^{37}$ (Eq. 11) to thermionic emission data from graphene based structures. Recent studies ${ }^{5-57}$ on graphene based Schottky barrier junction (lightly doped graphene with metal junction) show prospect of applying the equation in which the external voltage induced diode current density $J$ is governed by the saturation current density $\left(J_{0}\right)$. Also, $J_{0}$ is thought to be of thermionic origin and according to various authors different forms are given according to Javadi et al. ${ }^{57}$ in Eq. (12).

$$
J_{0}=C_{G}\left(q \phi_{B}\right) v_{\perp}^{*} \operatorname{Texp}\left(-\frac{q \phi_{B}}{k_{B} T}\right)
$$

where $C_{G}$ is a constant related to thermionic property of graphene and is shown to be $0.06 \mathrm{C} / \mathrm{eVcm}^{3} \mathrm{~K}^{57}$; $\phi_{B}$ is the Schottky barrier height, and $v_{\perp}$ is the out of plane electron velocity.

Consequently Sinha and $\mathrm{Lee}^{56}$, stated saturation current density $\left(J_{0}\right)$ as:

$$
J_{0}=A^{*} T^{2} \exp \left(-\frac{q \phi_{B}}{k_{B} T}\right)
$$

Kalita et al. ${ }^{55}$ gave the relation for saturation current density in graphene-silicon Schottky barrier diode same as in Eq. (13). However, none of these authors gave the experimental $J_{0}$ against $\mathrm{T}$ data and for the $J$ against $V$ graphs 
temperature (T) was not mentioned by Kalita et al. ${ }^{55}$ and Sinha and Lee ${ }^{56}$. In addition, Javedi et al. ${ }^{57}$ however mentioned temperature for their $J$ against $V$ (external voltage) curves but it is hard to extract the $J_{0}$ against T data which ideally should be external voltage independent. Thus, more experiments on such diodes are needed to extract $J_{0}$ against $\mathrm{T}$ data on which our model (MRDE) can be conveniently tested, whether it supports or fails. It would be an interesting field of research.

\section{Conclusion}

This study has discussed various models, including the present work results, and compared them in fitting the experimental results of thermionic emission current density versus temperature data for 2D graphene. We have given detailed reasons for the problems associated with a perfect physics model that should explain the massless nature of thermionic electron for motion in the 2D plane of graphene, finite mass, and discrete quantized energy levels for motion along the $z$-direction (thermionic emission). The study presents how various models failed to take care of these essential facts and the generation of momentum components along $z$-direction of thermionic electrons. In this paper, we have given a brief explanation of why a 3D dimensional model for thermionic emission from a 2D graphene may be appropriate, especially in the absence of a perfect physics model in literature, and we have considered very detailed comparisons of the previous models with our model that was not reported by us earlier.

This study also reveals how the Richardson Dushman mode ${ }^{49}$ fails to harmonize the experimental and theoretical results of current density vs $\mathrm{T}$ with published works. Also, Wei et al. model ${ }^{52}$, which was discussed qualitatively, is too difficult to handle. It would require electrons tunnelling through barriers of different widths and with varying times of relaxation (for tunnelling probabilities). Moreover, it is not easy to model the tunnelling times because of variation with quantized energies ${ }^{51}$. Thus, we observe a real challenge in formulating the current density-temperature relation of 2D graphene, considering the energy dispersion in the 2D plane and the discrete energy levels in the direction normal to the $2 \mathrm{D}$ plane of graphene.

Received: 22 February 2020; Accepted: 29 October 2021

Published online: 18 November 2021

\section{References}

1. Khoshaman, A. H., Fan, H. D. E., Koch, A. T., Sawatzky, G. A. \& Nojeh, A. Thermionics, thermoelectrics, and nanotechnology: New possibilities for old ideas. IEEE Nanotechnol. Mag. 8, 4-15 (2014).

2. Wang, M. S., Wang, J. Y. \& Peng, L.-M. Engineering the cap structure of individual carbon nanotubes and corresponding electron field emission characteristics. Appl. Phys. Lett. 88, 243108 (2006).

3. Schwede, J. W. et al. Photon-enhanced thermionic emission from heterostructures with low interface recombination. Nat. Commun. 4, 1576 (2013).

4. McCarthy, P. T., Reifenberger, R. G. \& Fisher, T. S. Thermionic and photo-excited electron emission for energy-conversion processes. Front. Energy Res. 2, 1-15 (2014) https://doi.org/10.3389/fenrg.2014.00054.

5. Westover, T. L., Franklin, A. D., Cola, B. A., Fisher, T. S. \& Reifenberger, R. G. Photo- and thermionic emission from potassiumintercalated carbon nanotube arrays. J. Vac. Sci. Technol. B Nanotechnol. Microelectron. Mater. Process. Meas. Phenom. 28, 423-434 (2010).

6. Trucchi, D. M. \& Melosh, N. A. Electron-emission materials: Advances, applications, and models. MRS Bull. 42, 448-492 (2017) https://doi.org/10.1557/mrs.2017.142.

7. Olawole, O. C. \& De, D. K. Theoretical studies of thermionic conversion of solar energy with graphene as emitter and collector. J. Photonics Energy 8, 1 (2018).

8. Olawole, O. C., De, D. K. \& Oyedepo, S. O. Energy dynamics of solar thermionic power conversion with emitter of graphene. Proc. SPIE 9932, (2016).

9. Geim, A. K. \& Novoselov, K. S. The rise of graphene. Nat. Mater. 6, 183-191 (2007).

10. Castro Neto, A. H., Guinea, F., Peres, N. M. R., Novoselov, K. S. \& Geim, A. K. The electronic properties of graphene. Rev. Mod. Phys. 81, 109-162 (2009).

11. Akinwande, D. et al. A review on mechanics and mechanical properties of 2D materials-Graphene and beyond. Extrem. Mech. Lett. 13, 42-77 (2017).

12. Proctor, J. Graphene is thin, but not infinitely so. Physics 12, 104 (2019).

13. Lin, Y-J \& Zeng, J-J. Tuning the work function of graphene by ultraviolet irradiation. Appl. Phys. Lett. 102, 183120 (2013) https:// doi.org/10.1063/1.4804289.

14. Kwon, K. C., Choi, K. S., Kim, B. J., Lee, J.-L. \& Kim, S. Y. Work-function decrease of graphene sheet using alkali metal carbonates. J. Phys. Chem. C 116, 26586-26591 (2012).

15. Naghdi, S., Sanchez-Arriaga, G. \& Rhee, K. Y. Tuning the work function of graphene toward application as anode and cathode. J. Alloys Compd. 805, 1117-1134 (2019).

16. Mishra, N., Boeckl, J., Motta, N. \& Iacopi, F. Graphene growth on silicon carbide: A review. Phys. Status Solidi 213, 2277-2289 (2016).

17. Sun, Y. W. et al. 3D strain in 2D materials: To what extent is monolayer graphene graphite?. Phys. Rev. Lett. 123, 135501 (2019).

18. Richardson, O. W. L. I. Some applications of the electron theory of matter. Lond. Edinb. Dublin Philos. Mag. J. Sci. 23, 594-627 (1912).

19. Richardson, O. W. Electron emission from metals as a function of temperature. Phys. Rev. 23, 153-155 (1924).

20. Liang, S.-J. \& Ang, L. K. Electron thermionic emission from graphene and a thermionic energy converter. Phys. Rev. Appl. 3, 014002 (2015).

21. Kribus, A. \& Segev, G. Solar energy conversion with photon-enhanced thermionic emission. J. Opt. 18, 073001 (2016).

22. Schwede, J. W. et al. Photon-enhanced thermionic emission for solar concentrator systems. Nat. Mater. 9, 762-767 (2010).

23. Modinos, A. Field, thermionic, and secondary electron emission spectroscopy. J. Colloid Interface Sci. (Springer Science +Business New York. 1984). https://doi.org/10.1007/978-1-4757-1448-7.

24. Nemanich, R. J. et al. Thermionic and field electron emission devices from diamond and carbon nanostructures. In $20103 \mathrm{rd}$ International Nanoelectronics Conference (INEC) 56-57 (IEEE, 2010). https://doi.org/10.1109/INEC.2010.5424519.

25. Murakami, K. et al. Electron emission properties of graphene-oxide-semiconductor planar-type electron emission devices. J. Vac. Sci. Technol. B Nanotechnol. Microelectron. Mater. Process. Meas. Phenom. 36, 02C110 (2018).

26. Giubileo, F. et al. Field emission from carbon nanostructures. Appl. Sci. 8, 526 (2018). 
27. Sun, S., Ang, L. K., Shiffler, D. \& Luginsland, J. W. Klein tunnelling model of low energy electron field emission from single-layer graphene sheet. Appl. Phys. Lett. 99, 013112 (2011).

28. Meir, S., Stephanos, C., Geballe, T. H. \& Mannhart, J. Highly-efficient thermoelectronic conversion of solar energy and heat into electric power. J. Renew. Sustain. Energy 5, 043127 (2013).

29. Khalid, K. A. A., Leong, T. J. \& Mohamed, K. Review on thermionic energy converters. IEEE Trans. Electron Devices 63, 2231-2241 (2016).

30. Xiao, G. et al. Thermionic energy conversion for concentrating solar power. Appl. Energy 208, 1318-1342 (2017)

31. Lee, J. H. et al. Microfabricated silicon carbide thermionic energy converter for solar electricity generation. In 2012 IEEE 25th International Conference on Micro Electro Mechanical Systems (MEMS) 1261-1264 (IEEE, 2012). https://doi.org/10.1109/MEMSYS. 2012.6170386.

32. Goodale, D. B., Reagan, P., Miskolczy, G., Lieb, D. \& Huffman, F. N. Combustion performance of CVD silicon carbide thermionic diodes. In: Energy to the 21st Century; Proceedings of the Fifteenth Intersociety Energy Conversion Engineering Conference 2095-2097 (1980).

33. Giannazzo, F. Insight into the mechanisms of chemical doping of graphene on silicon carbide. Nanotechnology 27, 072502 (2016).

34. Ahmad, I., Yazdani, B. \& Zhu, Y. Recent advances on carbon nanotubes and graphene. Nanomaterials 5, 90-114 (2015). https:// doi.org/10.3390/nano5010090.

35. Wei, Z. et al. Nanoscale tunable reduction of graphene oxide for graphene electronics. Science 328, 1373-1376 (2010).

36. Craciun, M. F., Russo, S., Yamamoto, M. \& Tarucha, S. Tuneable electronic properties in grapheme. Nano Today https://doi.org/ 10.1016/j.nantod.2010.12.001 (2011)

37. De, D. K. \& Olawole, O. C. A three-dimensional model for thermionic emission from graphene and carbon nanotube. J. Phys. Commun. 3, 015004 (2019)

38. Olukunle, O. C. \& De, D. K. Thermo-electronic solar power conversion with a parabolic concentrator. J. Semicond. 37, 024002 (2016).

39. De, D. K. \& Olawole, O. C. Modified Richardson-Dushman equation and modeling thermionic emission from monolayer graphene. Proc. SPIE 9927, 99270E (2016).

40. De, K. D. \& Olukunle, O. C. Graphene for thermoelectronic solar energy conversion. Proceedings of SPIE-The International Society for Optical Engineering 10368, 17 (2017).

41. Fowler, R. H. \& Nordheim, L. Electron emission in intense electric fields. Phys. Rev. Proc. Nat. Ac. Sci. Roy. Soc. Proc. Z. F. Phys. 119, 173-181 (1928).

42. Becker, J. A. Thermionic electron emission and adsorption part I. Thermionic emission. Rev. Mod. Phys. 7, 95-128 (1935).

43. Liang, S.-J., Liu, B., Hu, W., Zhou, K. \& Ang, L. K. Thermionic energy conversion based on graphene van der Waals heterostructures. Sci. Rep. 7, 46211 (2017).

44. Zhu, F. et al. Heating graphene to incandescence and the measurement of its work function by thermionic emission method. Nano Res. 7, 553-560 (2014). https://doi.org/10.1007/s12274

45. Misra, S., Upadhyay Kahaly, M. \& Mishra, S. K. Thermionic emission from monolayer graphene, sheath formation and its feasibility towards thermionic converters. J. Appl. Phys. 121, 065102 (2017).

46. Ang, Y. S., Chen, Y., Tan, C. \& Ang, L. K. Generalized high-energy thermionic electron injection at graphene interface. Phys. Rev. Appl. 12, 014057 (2019).

47. Yuan, H. et al. Back-gated graphene anode for more efficient thermionic energy converters. Nano Energy 32, 67-72 (2017).

48. Wanke, R. et al. Magnetic-field-free thermoelectronic power conversion based on graphene and related two-dimensional materials. J. Appl. Phys. 119, 244507 (2016).

49. Richardson, O. W. Thermionic phenomena and the laws which govern them (1928).

50. Khatoon, S. A., Ansari, M. M. \& Ashraf, S. Effect of temperature-dependent work function and Fermi energy on thermionic emission current density in graphene. In AIP Conference Proceedings 1953, 030239 (AIP Publishing LLC, 2018).

51. Wei, X., Wang, S., Chen, Q. \& Peng, L. Breakdown of Richardson's law in electron emission from individual self-joule heated carbon nanotubes. Sci. Rep. 4, 1-5 (2014).

52. Wei, X., Chen, Q. \& Peng, L. Electron emission from a two-dimensional crystal with atomic thickness. AIP Adv. 3, 042130 (2013).

53. Kim, H. \& Lee, J.-K. Generalized thermionic emission for arbitrary dimension. J. Korean Phys. Soc. 74, 701-706 (2019).

54. Yoon, H. et al. Measurement of collective dynamical mass of Dirac fermions in graphene. Nat. Nanotechnol. 9, 594-599 (2014).

55. Kalita, G., Hirano, R., Ayhan, M. E. \& Tanemura, M. Fabrication of a Schottky junction diode with direct growth graphene on silicon by a solid phase reaction. J. Phys. D. Appl. Phys. 46, 455103 (2013).

56. Sinha, D. \& Lee, J. U. Ideal graphene/silicon schottky junction diodes. Nano Lett. 14, 4660-4664 (2014).

57. Javadi, M., Noroozi, A. \& Abdi, Y. Kinetics of charge carriers across a graphene-silicon schottky junction. Phys. Rev. Appl. 14, 064048 (2020).

58. Liu, P. et al. Thermionic emission and work function of multiwalled carbon nanotube yarns. Physical Review B. 73(23), (2006). https://doi.org/10.1103/PhysRevB.73.235412.

59. Wei Y. et al. Comparative studies of multiwalled carbon nanotube sheets before and after shrinking. Physical Review B. 76(4), (2007). https://doi.org/10.1103/PhysRevB.76.045423

60. Liu P. et al. Measuring the work function of carbon nanotubes with thermionic method. Nano Letters 8(2), 647-651 (2008). https:// doi.org/10.1021/nl0730817

61. Huang S. et al. High efficiency and non-Richardson thermionics in three dimensional Dirac materials. Appl. Phys. Lett. 111(18) 183902, (2017). https://doi.org/10.1063/1.5006277

\section{Acknowledgements}

The authors gratefully appreciate the facilities provided by the Covenant University to carry out the reported research work. The authors thank Dr. Peng Liu and Professor Kaili Jiang for providing them with original experimental data. F.I.E. acknowledges the support received from the Africa Centre of Excellence for Sustainable Power and Energy Development (ACE-SPED), University of Nigeria, Nsukka.

\section{Author contributions}

O.C.O., D.K.D. wrote the main manuscript, S.O.O., F.I.E. wrote the introduction and prepared Figs. 1 and 2. O.C.O. prepared Fig. 3 and Table 1. All authors reviewed the manuscript.

\section{Competing interests}

The authors declare no competing interests.

\section{Additional information}

Correspondence and requests for materials should be addressed to O.C.O. 
Reprints and permissions information is available at www.nature.com/reprints.

Publisher's note Springer Nature remains neutral with regard to jurisdictional claims in published maps and institutional affiliations.

(c) (i) Open Access This article is licensed under a Creative Commons Attribution 4.0 International License, which permits use, sharing, adaptation, distribution and reproduction in any medium or format, as long as you give appropriate credit to the original author(s) and the source, provide a link to the Creative Commons licence, and indicate if changes were made. The images or other third party material in this article are included in the article's Creative Commons licence, unless indicated otherwise in a credit line to the material. If material is not included in the article's Creative Commons licence and your intended use is not permitted by statutory regulation or exceeds the permitted use, you will need to obtain permission directly from the copyright holder. To view a copy of this licence, visit http://creativecommons.org/licenses/by/4.0/.

(C) The Author(s) 2021 